\title{
Influencia del cambio climático en los escenarios del futuro sobre plantaciones de Eucalipto ${ }^{1}$
}

\author{
Influence of climate change on future scenarios on Eucalyptus plantations
}

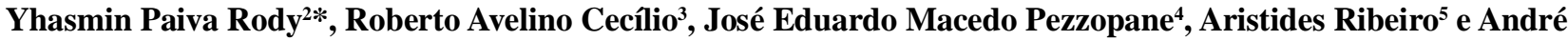 \\ Quintão de Almeida ${ }^{6}$
}

\begin{abstract}
RESUMEN - El género Eucalyptus presenta gran potencial con fines comerciales y es la especie de árbol más plantada en Brasil. Las perspectivas del cambio climático en las proyecciones futuras del IPCC (Intergovernmental Panel on Climate Change) indican que se pueden producir pérdidas significativas en las actividades agrícolas y forestales. El objetivo de este estudio fue determinar las zonas aptas para plantaciones de Eucalyptus grandis, sobre la base de datos climatológicos y de relieve en los escenarios futuros. El trabajo fue desarrollado en la provincia del Espirito Santo (ES-Brasil). Fueron utilizadas las normales de clima obtenidas de estaciones pluviométricas. El balance de agua se calculó por el método de Tornthwaite y Mather. Se estimó la temperatura con base en los datos de latitud y altitud. Se utilizaron los datos SRTM para obtener la altitud y la declividad para las zonas de mecanización agrícola. Para la interpolación de los datos se utilizó el método del inverso del cuadrado de la distancia. Los datos de escenarios futuros se obtuvieron del modelo HADCM3 del IPCC en escenarios A (pesimista) y B (optimista) estimados para los años de 2020, 2050 y 2080. Se estima que las zonas aptas para plantaciones se redujeron en ambos escenarios, llegando a la reducción de 71,7\% y 82,5\% en el escenario A y B, respectivamente para el año de 2080. El déficit de agua actuó como un factor limitante en la plantación. Deben llevarse a cabo nuevos estudios relacionando la aptitud de la especie en el contexto del cambio climático.
\end{abstract}

Palabras-clave: Eucalyptus grandis. Zonificación ambiental. Datos topográficos. IPCC.

\begin{abstract}
The genus Eucalyptus presents a large commercial potential and it is the arboreal species most planted in Brazil. Perspectives of climate change in the future projections of the IPCC (Intergovernmental Panel on Climate Change) indicate that productivity losses may occur in diverse agricultural and forestry crops. The objective of this study was to determine the zones apt for planting of eucalyptus based on relief and climate data for current and future scenarios. The study was developed in the state of Espírito Santo, Brazil, and normal climatological data was obtained from rain gauges. The water balance was calculated by the Tornthwaite and Mather methodology. Temperature was estimated using data of latitude and altitude. Data from the SRTM were used to obtain the altitude and zone of agricultural mechanization, based on slopes. For interpolation of the data, the inverse square distance method was used. The future data were estimated by the HADCM3 model of the IPCC in scenarios: A (pessimistic) and B (optimistic) for the years of 2020, 2050 and 2080. It is estimated that the zones suitable for planting are reduced in both scenarios, diminishing by $71.1 \%$ and $82 \%$ for scenarios A and B, respectively in the year 2080 . The water deficit acted as a limiting factor for planting. Due to the complexity of interactions of biotic and abiotic factors, new capability studies should be performed in the context of climate change.
\end{abstract}

Key words: Eucalyptus grandis. Environmental zoning. Topographic data. IPCC.

\footnotetext{
*Autor para correspondência

${ }^{1}$ Recebido para publicação em 28/01/2011; aprovado em 17/02/2012

Pesquisa realizada com o apoio do Conselho Nacional de Desenvolvimento Científico e Tecnológico - CNPq

${ }^{2}$ Departamento de Engenharia Agrícola, Universidade Federal de Viçosa/UFV, Viçosa-MG, Brasil, yhasmin@biomodel.ufv.br

${ }^{3}$ Departamento de Engenharia Florestal, Centro de Ciências Agrárias/UFES, Alegre-ES, Brasil, racecilio@yahoo.com.br

${ }^{4}$ Departamento de Engenharia Florestal, Centro de Ciências Agrárias/UFES, Alegre-ES, Brasil, pezzopane@ reitoria.ufes.br

${ }^{5}$ Departamento de Engenharia Agrícola, Universidade Federal de Viçosa/UFV, Viçosa-MG, Brasil, ribeiro@ufv.br

${ }^{6}$ Unidade Acadêmica de Serra Talhada/Fazenda Saco/Universidade Federal Rural de Pernambuco/UFPE, Serra Talhada-PE, Brasil, andreqa@ gmail.com
} 


\section{INTRODUCCIÓN}

Las variables meteorológicas actúan directa e indirectamente en los procesos referentes al crecimiento de las plantas. La temperatura influye en procesos fisiológicos como la respiración, la transpiración, la división celular, la fotosíntesis (CUNHA et al., 2009) y las fenofases, tales como la senescencia y la emisión de nuevas hojas (MARTINS et al., 2007). La disponibilidad hídrica tiene una gran relación con la transpiración mediante el control de la abertura estomática y por consiguiente, con el proceso de difusión de vapor de agua entre la cavidad estomática y la atmósfera (CARNEIRO et al., 2008). Por estas razones debería realizarse una evaluación más rigurosa de estos elementos antes de comenzar en la silvicultura una plantación.

Una revisión del "Climate Change: The Scientific Basis" preparado por el Intergovernmental Panel on Climate Change (IPCC WORKING GROUPI, 2001), llegó a la conclusión de que la media mundial de evaporación, la precipitación acumulada y la intensidad de lluvias se aumentarán en gran medida en respuesta al creciente aumento del efecto invernadero en la atmósfera. Estudios recientes han demostrado que estos cambios podrían tener importantes repercusiones en la productividad de los cultivos (ASSAD et al., 2004; KADISHI et al., 2005; ROUNSEVELL et al., 2005; HAIM et al., 2008).

Estos impactos se deben a los cambios fotosintéticos, de respiración y a la reducción en el ciclo del cultivo. Sin embargo, todavía hay una serie de incertidumbres, dado que la productividad depende de una serie de factores biofísicos y socioeconómicos que son difíciles de cuantificar (EWERT et al., 2005).

Los efectos de algunas variables meteorológicas y sus interacciones son complejos, dinámicos y no lineales. Un cambio en las precipitaciones, por ejemplo, afecta la erosión del suelo y proporciona un crecimiento diferenciado de determinados cultivos agrícolas, por un cambio en la frecuencia frente a la severidad (PEZZOPANE et al., 2010). El impacto actual de las variables individuales y sus interacciones puede diferenciarse en el tiempo y geográficamente, pudiendo solamente ser evaluado adecuadamente en un nivel complejo del sistema. Los modelos (matemáticos de siembra) representan el número de agro-sistemas como instrumentos poderosos para ayudar a estos asuntos (LUO et al., 2005).

En el modelo HadCM3 de clima, desarrollado en 1998, se encuentran los componentes de circulación general entre la atmósfera-océano, pudiendo ser usado para predecir el futuro del clima. Este modelo fue uno de los más utilizados en el Tercer Informe de Evaluación del IPCC-WGI (2001). Otros detalles del modelo HadCM3 son descritos por Gordon et al. (2000).

A pesar de que a nivel internacional se presta mucha atención por la oportunidad de secuestro de carbono para mitigar el cambio climático, se presta poca atención al régimen de los sistemas modificados con la combinación de este tipo de sistemas (TRABUCCO et al., 2008). El conocimiento de los posibles efectos del cambio climático es muy importante para el sector forestal, ya que permite la elaboración de estrategias para minimizar daños futuros.

De lo expuesto con anterioridad, el objetivo de este estudio fue determinar las zonas aptas para la siembra de Eucalyptus grandis en el Estado de Espirito Santo (Brasil), utilizando datos meteorológicos y topográficos en los escenarios futuros, a través del modelo HadCM3.

\section{MATERIALES Y METODOS}

El estudio fue adelantado en el Estado de Espirito Santo-Brasil, situado entre las coordenadas $17^{\circ} 52^{\prime}$ y $21^{\circ} 17^{\prime}$ de latitud y $41^{\circ} 54^{\prime}$ y $39^{\circ} 38^{\prime}$ de longitud. El estado se encuentra en la parte oriental de la región sudeste de Brasil y su territorio está entre dos regiones naturales diferentes: la zona del litoral, que se extiende por $400 \mathrm{~km}$, y la zona de meseta. A lo largo de la costa atlántica se encuentra una franja de llanura que representa el $40 \%$ de la superficie total del estado, y en la medida que se entra hacia el interior, la meseta da lugar a una región montañosa, con altitudes por encima de $1000 \mathrm{~m}$ (CITYBRASIL, 2009). El clima es tropical húmedo, con temperatura promedio anual de $23^{\circ} \mathrm{C}$, y el volumen de precipitaciones es superior a $1.400 \mathrm{~mm}$ por año, concentrados principalmente en el verano.

Para este estudio, se utilizaron los datos de temperatura, precipitaciones y de déficit hídrico en la caracterización del clima. Por no tener una serie histórica de mediciones de temperatura del aire y estaciones meteorológicas, esta variable fue estimada por el modelo matemático desarrollado por Pezzopane et al. (2004), el cual fue ajustado para todo el estado, como se indica en la ecuación:

$$
\mathrm{y}_{1}=\beta_{0}+\beta_{1} \mathrm{~A} 1 \mathrm{t}+\beta_{2} \mathrm{Lat}
$$

donde: $\mathrm{Yi}=$ Temperatura del aire $\left({ }^{\circ} \mathrm{C}\right) ;$ Alt $=$ Altitud $(\mathrm{m})$; Lat $=$ latitud en grados decimales; $\beta 0, \beta 1$ y $\beta 2=$ coeficientes de la regresión ajustada para cada mes del año.

Se usaron las series históricas de lluvias recolectadas en puntos pluviométricos de la Agencia Nacional del Agua (ANA) con datos de la precipitación anual media, obtenidos en 86 puntos en el período comprendido entre 1976 y 2003 (Figura 1).

La disponibilidad hídrica fue determinada a partir de los resultados del balance hídrico climatológico promedio (THORNTHWAITE y MATTHER, 1955), calculados en función de la precipitación y temperatura promedio mensual. Se usó una capacidad de almacenamiento de agua en el suelo de $100 \mathrm{~mm}$. 
Figura 1 - Puntos Pluviométricos - Estado del Espirito Santo

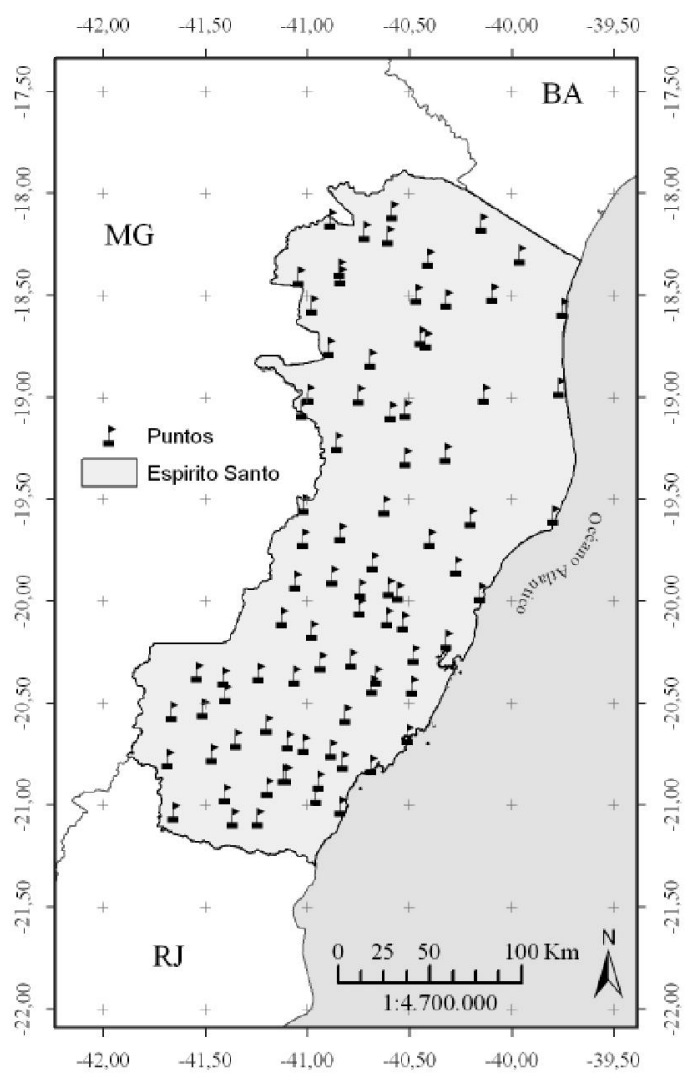

Fueron utilizados los datos de la proyección futura de la precipitación y temperatura, reportados por "The Special Report on Emission Scenarios" (SRES), estimados a partir del modelo global HadCM3. La elección del modelo se debe por permitir evaluar el efecto de la magnitud del cambio climático en las plantaciones forestales, además de la aplicación en distintos trabajos en el área agrícola (PARRY et al., 2004; BERRY et al., 2006; FADER et al., 2010).

El trabajo fue realizado en dos escenarios de emisiones: A2 y B2 previstos para los años 2020, 2050 y 2080. El escenario A2 es aquel escenario pesimista, en donde se considera un aumento poblacional creciente y sin adopción de medidas ambientales correctas, como por ejemplo, en relación a la disminución de la emisión de $\mathrm{CO}_{2}$ en el ambiente. El B2 es aquel escenario donde la concientización de la población es mayor y existen medidas de sustentabilidad ya empleadas.

La resolución espacial del modelo HadCM3 es de $2,5^{\circ}$ y $3,75^{\circ}$ (latitud y longitud), donde la precipitación es considerada como variable primaria, y su ocurrencia es basada en la distribución de longitudes de secuencias continuas de días secos y húmedos.

Con los nuevos valores de temperatura y precipitación mensual en los tres años futuros para cada escenario futuro (A2 y B2), se realizó nuevamente el balance hídrico.

En la espacialización de los datos climáticos, se utilizó el interpolador lineal Inverso de la Distancia Ponderada (IDW) con el exponente a la segunda potencia. El valor predicho es dado por un promedio ponderado, el cual utiliza el peso de los puntos de control más cercanos, ponderados por la inversa de la distancia elevada a un exponente $\mathrm{m}$. La representación matemática de esta función esta dada por la siguiente ecuación:

$$
\mathrm{Gi}=\frac{\sum_{\mathrm{i}=1}^{\mathrm{n}}\left(\frac{\mathrm{x}_{\mathrm{i}}}{\mathrm{d}_{\mathrm{i}}^{\mathrm{m}}}\right)}{\sum_{\mathrm{i}=1}^{\mathrm{n}}\left(\frac{1}{\mathrm{~d}_{\mathrm{i}}^{\mathrm{m}}}\right)}
$$

donde: $\mathrm{xi}=$ peso del $\mathrm{i}$-ésimo punto del muestreo; $\mathrm{di}=$ distancia euclidiana de i-ésimo punto del muestreo al punto actual; $\mathrm{m}=$ exponente de la función de la distancia euclidiana.

Se utilizó como Modelo Digital de Elevación (MDE) del Estado, los datos de "Shuttle Radar Topography Mission" (SRTM) de la "National Aeronautics and Space Administration" (NASA) proporcionada por Miranda y Coutinho (2009). Estos presentan una resolución espacial de $90 \mathrm{~m}$ y escala de 1: 250000 .

Para la realización de la zonificación de los sitios adecuados para la plantación de Eucalyptus grandis fueron considerados los aspectos para el buen desarrollo de la especie (Tabla 1), según las recomendaciones dadas en los trabajos realizados por Golfari (1975) y Nappo et al. (2005).

Tabla 1: Requisitos establecidos para la implantación de la especie trabajada. $\mathrm{P}=$ precipitación anual, $\mathrm{T}=$ temperatura media anual y D H = déficit hídrico anual

\begin{tabular}{ccccc}
\hline \multirow{2}{*}{ Especie } & \multicolumn{3}{c}{ Aspectos Climáticos } & Aspecto Topográfico \\
\cline { 2 - 5 } & $\mathrm{P}(\mathrm{mm})$ & $\mathrm{T}(\mathrm{oC})$ & $\mathrm{DH}(\mathrm{mm})$ & Altitud $(\mathrm{m})$ \\
\hline Eucalyptus grandis & $1000 \mathrm{a} 1800$ & $6 \mathrm{a} \mathrm{32}$ & 0 a 400 & $0-1200$ \\
\hline
\end{tabular}


Con los parámetros establecidos para la realización de la zonificación, fue realizado el cruce de las informaciones necesarias para la especie, con los parámetros determinados para cada año en cada uno de los escenarios.

\section{RESULTADOS Y DISCUSIÓN}

La Tabla 2 da a conocer la variación de la temperatura media anual y de la precipitación anual para una muestra de los puntos de la serie estudiada en los dos escenarios del modelo HadCM3. Se observa que el incremento estimado de la temperatura aumenta continuamente a lo largo de los años previstos en los escenarios A2 y B2. Adicionalmente, se encontró que los puntos que se encuentran cerca del nivel del mar (punto 1), unidos con aquellos en altitudes intermedias de $580 \mathrm{~m}$ (punto 17) y mayores con $1075 \mathrm{~m}$ (punto 24), tienen el mismo aumento de $0,7^{\circ}$ de temperatura para el año 2020 . La misma tendencia fue observa para los años de 2050 y 2080.

El bajo nivel de detalle del modelo, dado las variaciones de temperatura a diferentes altitudes, indica la necesidad de construir modelos regionales con mayor precisión y escalas. Rounsevell et al. (2005), proyectando cambios para el cultivo agrícola y de pastos, en los escenarios futuros, verificó que la baja escala trabajada no era sensible a los cambios climáticos a nivel regional.

Los dos primeros puntos de la Tabla 2, indican un cambio de $0,7{ }^{\circ} \mathrm{C}$ y $0,4{ }^{\circ} \mathrm{C}$, respectivamente, para la temperatura media estimada para el año 2020 en el escenario

Tabla 2 - Variación de las temperaturas medias anuales (T) y de la precipitación acumulada (P) para el escenarios A2 y B2 en relación con los datos actuales y localización geográfica de los puntos (Latitud - lat.; Longitud - Long; Altitud - Alt)

\begin{tabular}{|c|c|c|c|c|c|c|c|c|c|c|c|c|c|c|c|}
\hline \multirow{2}{*}{ Puntos } & \multirow{2}{*}{ Lat. } & \multirow{2}{*}{ Long. } & \multirow{2}{*}{ Alt. (m) } & \multicolumn{3}{|c|}{$\mathrm{T}\left({ }^{\circ} \mathrm{C}\right)-\mathrm{A} 2$} & \multicolumn{3}{|c|}{$\mathrm{T}\left({ }^{\circ} \mathrm{C}\right)-\mathrm{B} 2$} & \multicolumn{3}{|c|}{$\mathrm{P}(\mathrm{mm})-\mathrm{A} 2$} & \multicolumn{3}{|c|}{$\mathrm{P}(\mathrm{mm})-\mathrm{B} 2$} \\
\hline & & & & 2020 & 2050 & 2080 & 2020 & 2050 & 2080 & 2020 & 2050 & 2080 & 020 & 2050 & 2080 \\
\hline 1 & $-19,6$ & $-39,8$ & 3 & 0,7 & 2,3 & 3,4 & 0,8 & 1,8 & 3,4 & 47,2 & 44,3 & $-126,7$ & 27,2 & 10,7 & $-63,1$ \\
\hline 2 & $-21,0$ & $-40,8$ & 4 & 0,4 & 1,4 & 1,9 & 0,4 & 1,0 & 3,5 & 53,3 & $-16,4$ & $-125,5$ & $-55,3$ & $-79,9$ & $-108,7$ \\
\hline 3 & $-19,8$ & $-40,3$ & 58 & 0,7 & 2,3 & 3,4 & 0,8 & 1,8 & 3,5 & 47,2 & 44,3 & $-126,7$ & 27,2 & 10,7 & $-63,1$ \\
\hline 4 & $-20,9$ & $-40,9$ & 00 & 0,7 & 2,3 & 3,4 & 0,8 & 1,8 & 3,8 & 47,2 & 44,3 & $-126,7$ & 27,2 & 10,7 & $-63,1$ \\
\hline 5 & $-21,1$ & $-41,2$ & 120 & 0,4 & 1,4 & 1,9 & 0,4 & 1,0 & 3,5 & 53,3 & $-16,4$ & $-125,5$ & $-55,3$ & $-79,9$ & $-108,7$ \\
\hline 6 & $-19,2$ & $-40,9$ & 135 & 0,7 & 2,3 & 3,4 & 0,8 & 1,8 & 3,3 & 47,2 & 44,3 & $-126,7$ & 27,2 & 10,7 & $-63,1$ \\
\hline 7 & $-19,1$ & $-40,6$ & 170 & 0,7 & 2,3 & 3,4 & 0,8 & 1,8 & 3,3 & 47,2 & 44,3 & $-126,7$ & 27,2 & 10,7 & $-63,1$ \\
\hline 8 & $-20,7$ & $-41,3$ & 180 & 0,7 & 2,2 & 3,9 & 0,8 & 1,9 & 3,6 & 112,2 & 191,7 & 255,8 & 66,1 & 75,7 & 161,6 \\
\hline 9 & $-18,8$ & $-40,9$ & 192 & 0,7 & 2,3 & 3,4 & 0,8 & 1,8 & 3,2 & 47,2 & 44,3 & $-126,7$ & 27,2 & 10,7 & $-63,1$ \\
\hline 10 & $-19,9$ & $-41,1$ & 250 & 0,7 & 2,3 & 3,4 & 0,8 & 1,8 & 3,5 & 47,2 & 44,3 & $-126,7$ & 27,2 & 10,7 & $-63,1$ \\
\hline 11 & $-18,1$ & $-40,6$ & 290 & 0,7 & 2 & 2,9 & 0,6 & 1,4 & 3 & 67,8 & 90,2 & $-109,7$ & 47,1 & 156,5 & $-21,6$ \\
\hline 12 & $-20,1$ & $-41,1$ & 0 & 0,7 & 2,3 & 3,4 & 0,8 & 1,8 & 3,7 & 47,2 & 44,3 & $-126,7$ & 27,2 & 10,7 & $-63,1$ \\
\hline 13 & $-20,5$ & $-41,5$ & 380 & 0,6 & 2,2 & 3,9 & 0,8 & 1,9 & 3,7 & 112,2 & 191,7 & 255,8 & 66,1 & 75,6 & 161,6 \\
\hline 14 & $-18,2$ & $-40,6$ & 400 & 0,7 & 2 & 2,9 & 0,6 & 1,4 & 3,1 & 67,8 & 90,2 & $-109,7$ & 47,1 & 156,5 & $-21,6$ \\
\hline 15 & $-20,4$ & $-40,7$ & 452 & 0,7 & 2,3 & 3,4 & 0,8 & 1,8 & 3,9 & 47,2 & 44,3 & $-126,7$ & 27,2 & 10,7 & $-63,1$ \\
\hline 16 & $-20,4$ & $-40,7$ & 5 & 0,7 & 2 & 3,4 & 0,8 & 1,8 & 3,9 & 47,2 & 44,3 & $-126,7$ & 27,2 & 10,7 & $-63,1$ \\
\hline 17 & $-20,4$ & $-41,4$ & 580 & 0,7 & 2,2 & 3,9 & 0,8 & 1,9 & 3,7 & 112,2 & 191,7 & 255,8 & 66,1 & 75,7 & 161,6 \\
\hline 18 & $-20,9$ & $-41,4$ & 600 & 0,7 & 2,2 & 3,9 & 0,8 & 1,9 & 3,8 & 112,2 & 191,7 & 255,8 & 66,1 & 75,7 & 161,6 \\
\hline 19 & $-20,3$ & $-40,8$ & 640 & 0,7 & 2,3 & 3,4 & 0,8 & 1,8 & 3,9 & 47,2 & 44,3 & $-126,7$ & 27,2 & 10,7 & $-63,1$ \\
\hline 20 & $-19,9$ & $-40,7$ & 690 & 0,7 & 2,3 & 3,4 & 0,8 & 1,8 & 3,9 & 47,2 & 44,3 & $-126,7$ & 27,2 & 10,7 & $-63,1$ \\
\hline 21 & $-20,7$ & $-41,1$ & 772 & 0,7 & 2,3 & 3,4 & 0,8 & 1,8 & 3,8 & 47,2 & 44,3 & $-126,7$ & 27,2 & 10,7 & $-63,1$ \\
\hline 22 & $-20,3$ & $-40,9$ & 804 & 0,7 & 2,3 & 3,4 & 0,8 & 1,8 & 4 & 47,2 & 44,3 & $-126,7$ & 27,2 & 10,7 & $-63,1$ \\
\hline 23 & $-20,1$ & $-41,0$ & 940 & 0,7 & 2,3 & 3,4 & 0,8 & 1,8 & 4 & 47,2 & 44,3 & $-126,7$ & 27,2 & 10,7 & $-63,1$ \\
\hline 24 & $-20,4$ & $-41,1$ & 1075 & 0,7 & 2,3 & 3,4 & 0,8 & 1,8 & 4,1 & 47,2 & 44,3 & $-126,7$ & 27,2 & 10,7 & $-63,1$ \\
\hline
\end{tabular}

El estado de Espirito Santo es actualmente apto para la plantación de especies de Eucalyptus grandis en un 97,2\% de su superficie, de acuerdo con los parámetros climáticos y topográficos evaluados (Figura 2). Es importante resaltar que para realizar un análisis mas completo, los parámetros edáficos deben ser considerados, lo cual no fue posible tener en cuenta en este trabajo 
A2. Estos puntos se encuentran en altitudes similares, siendo la latitud un factor que contribuye a este cambio, ya que los dos se encuentran en el extremo norte y sur del estado.

La variación en las precipitaciones anuales no sigue la misma tendencia de la temperatura media con el pasar de los años. Del año 2050, y más significativamente a partir del 2080, se puede estimar una disminución en relación con la precipitación actual.

Las áreas menos aptas para el cultivo se encuentran en el surdeste del estado, en la "Serra de Caparaó", que se encuentra en el "Pico da Bandeira", que es el tercer punto más alto de Brasil, con una altura de $2800 \mathrm{~m}$. En la parte central al oeste del estado, el área no apta se debe a la presencia de precipitaciones por debajo de los $1000 \mathrm{~mm}$ requeridos por la especie.

Los Programas de fomento Forestal implementados por las empresas de celulosa y asociaciones del gobierno, están incentivando a los propietarios de tierras a la plantación de Eucalipto en este Estado. Se estima en la actualidad que hay alrededor de 3.751 .867 ha con plantaciones de este género (ABRAF, 2007).

Figura 2 - Áreas aptas/inaptas para la plantación de Eucalyptus grandis en el Estado do Espirito Santo en la actualidad

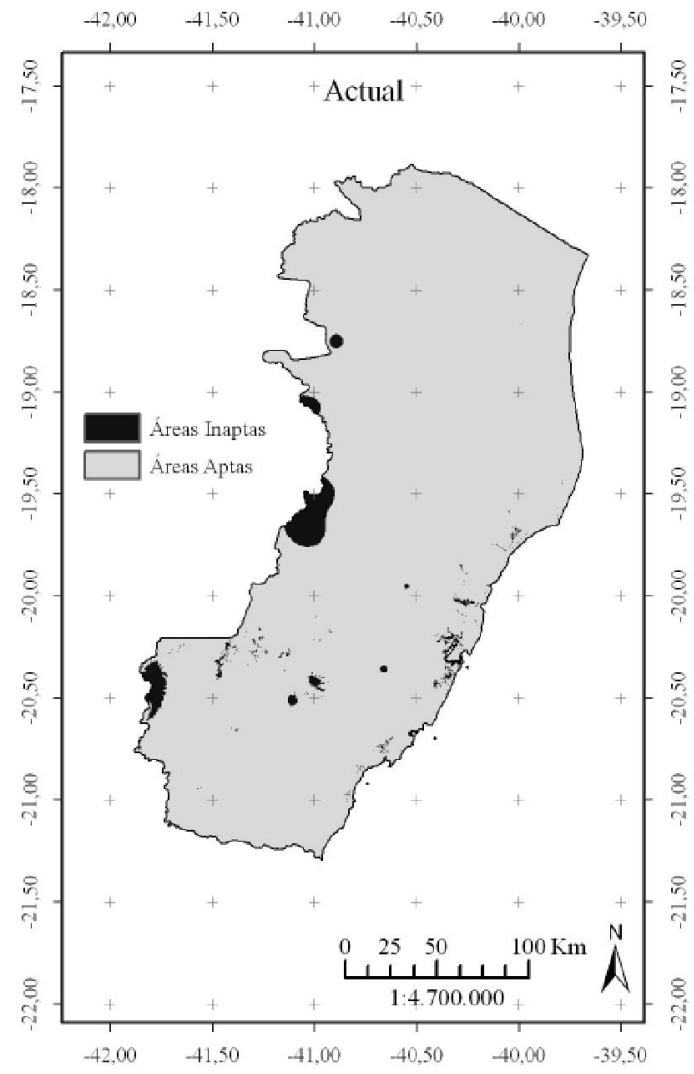

Para el año 2020, se estima que el 58,20\% de la superficie total del estado sea apta para la plantación de Eucalyptus grandis en el escenario A2. De acuerdo con el escenario B2, el 62,5\% de la superficie es apta para este tipo de especies forestales.

La Figura 4, indica el aumento considerable de áreas no aptas en los dos escenarios en el año de 2050. Se verifica que las áreas que ocupan la porción central hasta el norte del estado, son prácticamente todas desfavorables para la plantación de esta especie forestal. Se estima que el 35 y $38 \%$, para los escenarios A2 y B2 respectivamente, son aptas para la plantación, indicando una disminución considerable de áreas en relación al año 2020.

En el año 2080, según las estimativas del modelo, el 17 y $27,5 \%$ de las zonas pueden ser plantadas con Eucalyptus grandis, de acuerdo a los escenarios A2 y B2. Haim et al. (2008) encontraron que cuando se utilizó el modelo HadCM3 en el período 2070 a 2100, el rendimiento de la plantación de algodón se redujo en los dos escenarios analizados (A2 y B2).

Se observa que para los tres años estimados, el escenario A2 subestimó la cantidad de áreas empleadas en la plantación, en relación al escenario B2. La cadena causal de los cambios del clima y sus impactos son básicamente los siguientes: presencia de las emisiones antropogénicas, que son modeladas a partir de escenarios de la alteración poblacional, de producción, y de uso de energía, como también de modelos económicos.

Analizando los factores climáticos que definieron un gran porcentaje de las áreas no aptas para la siembra en los años siguientes con respecto al tiempo actual, se verificó que la temperatura no incidió en ninguno de los años para los dos escenarios trabajados, ya que la franja de tolerancia para el Eucalyptus grandis es amplia. A diferencia de lo anterior, Assad et al. (2004) encontraron un aumento de 61,6 y 85,9\% en las áreas no aptas para Minas Gerais-Brasil, simulando un aumento de $1^{\circ} \mathrm{C}$ en la temperatura, trabajando con un cultivo de café.

Existe una clara diferencia en el nivel de restricción en relación a las dos variables climatológicas expuestas. Son consideradas áreas aptas para la plantación el 71,6\% impuesto por el déficit hídrico y $8 \%$ para la precipitación en el escenario más optimista (B2). Evaluando los puntos estudiados, el déficit hídrico se presentó por encima del límite considerado en este trabajo para el buen desempeño de la especie en 45,5 y $60,5 \%$ respectivamente, para los escenarios A2 y B2 para el año 2080.

En acuerdo con estos resultados, Fuher (2003) cita que los mayores limitantes para la producción en el futuro serán la deficiencia de agua y nutrientes. Esta premisa se supone ya que los efectos en el aumento de la productividad con el aumento de $\mathrm{CO}_{2}$, pueden ser compensados por el aumento de la temperatura. 
Figura 3 - Áreas aptas/inaptas para la plantación de Eucalyptus grandis en el escenario A2 y B2 en 2020
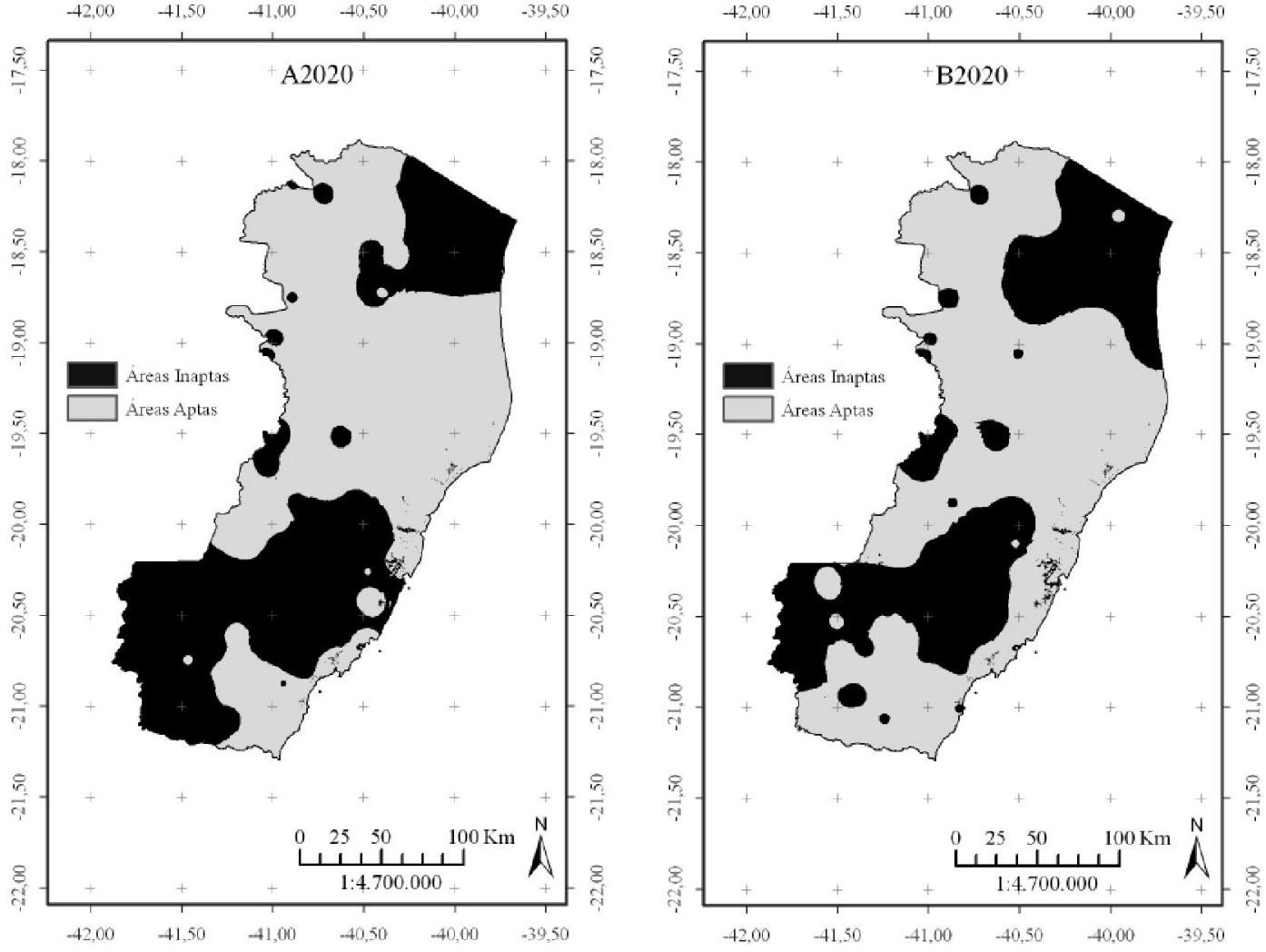

Figura 4 - Áreas aptas/inaptas para la plantación de Eucalyptus grandis en el escenario A2 y B2 en 2050
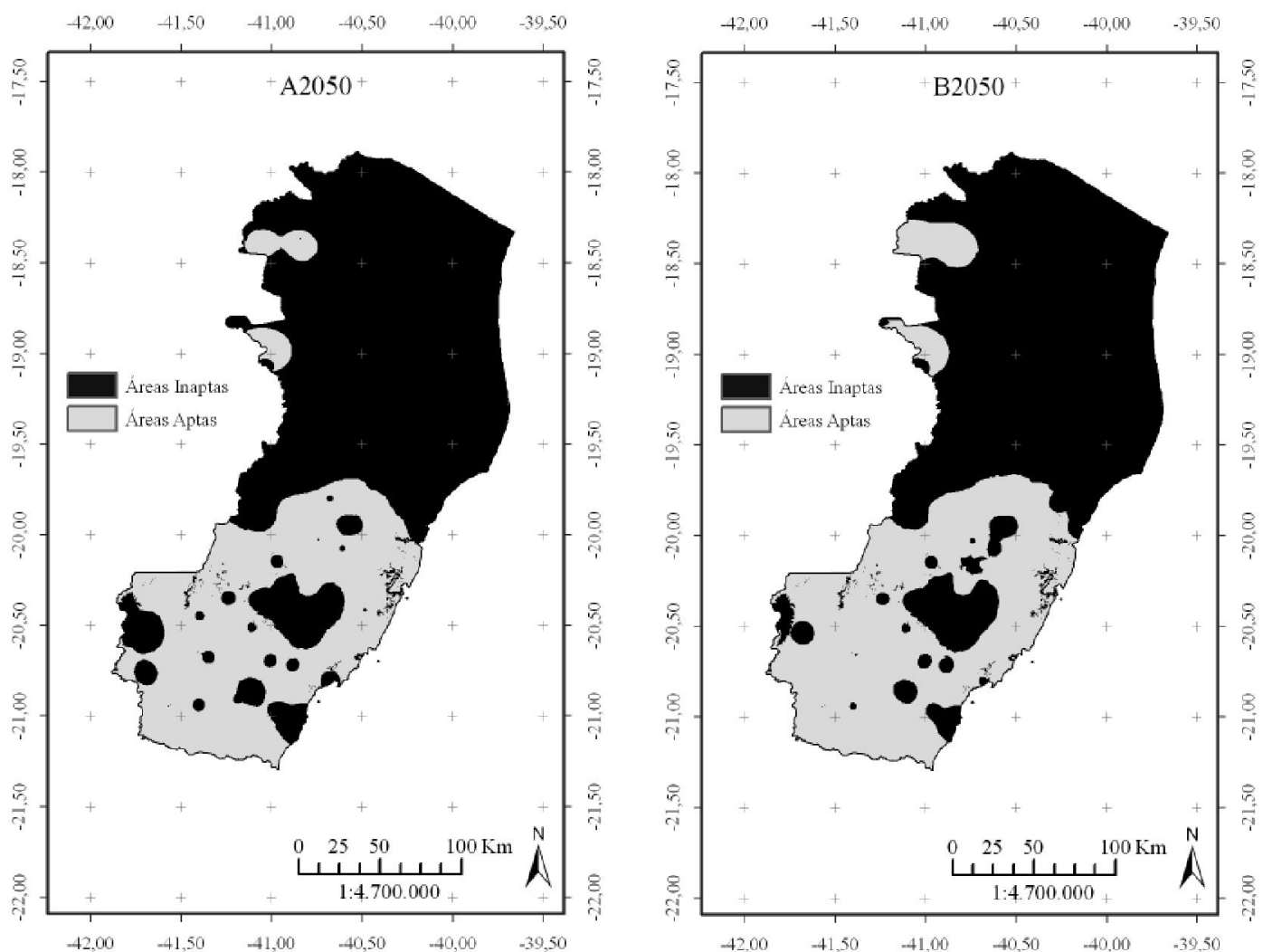
Figura 5 - Zonas adecuadas / inadecuadas para la plantación de Eucalyptus grandis en el escenario A2 y B2 en 2080

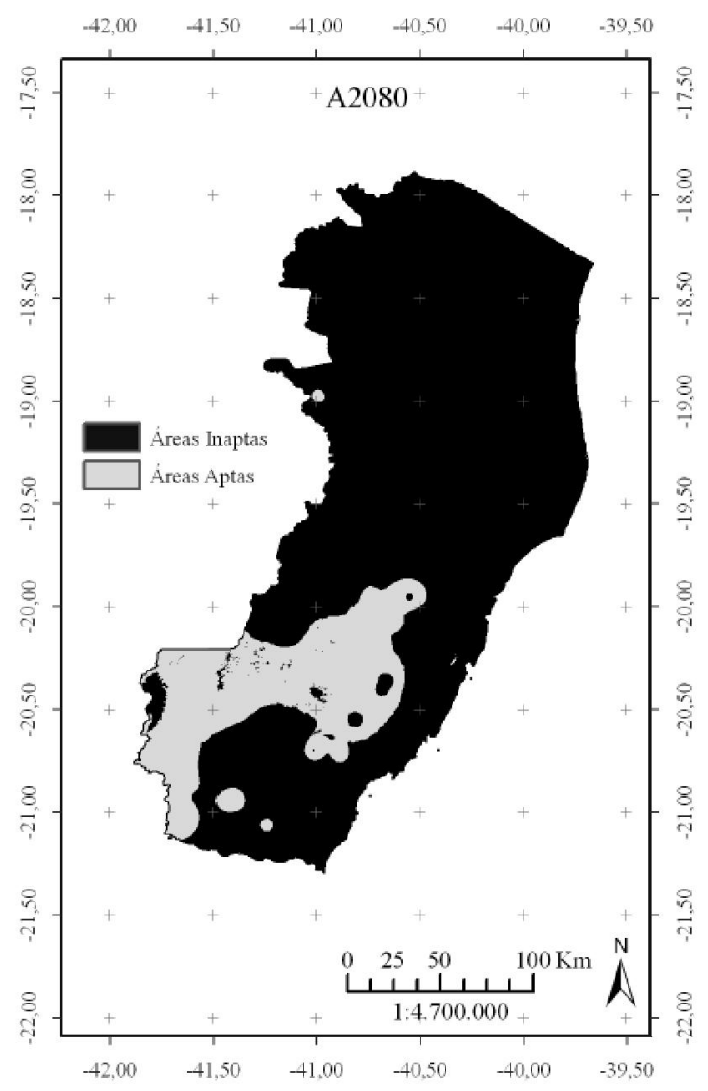

\section{CONCLUSIONES}

1. El bajo nivel de detalle de un modelo global pone en evidencia la necesidad de implementar modelos regionales con grandes escalas;

2. El estado de Espirito Santo es apropiado en la actualidad en casi toda su superficie para la plantación de Eucalyptus grandis de acuerdo con los parámetros de clima y topografía evaluados. Parece que durante tres años estimados, el escenario A2 ha subestimado las zonas de plantaciones en relación con B2;

3. Los resultados sugieren que la variable déficit de agua tiene una gran contribución al aumentar las zonas no aptas para la plantación a lo largo de los años estimados.

\section{REFERENCIAS}

ABRAF - Associação Brasileira Florestal. Anuário Estatístico da ABRAF 2008 - Ano Base 2007. Disponível em: http://www. abraflor.org.br/. Acesso em: 15/8/2010.

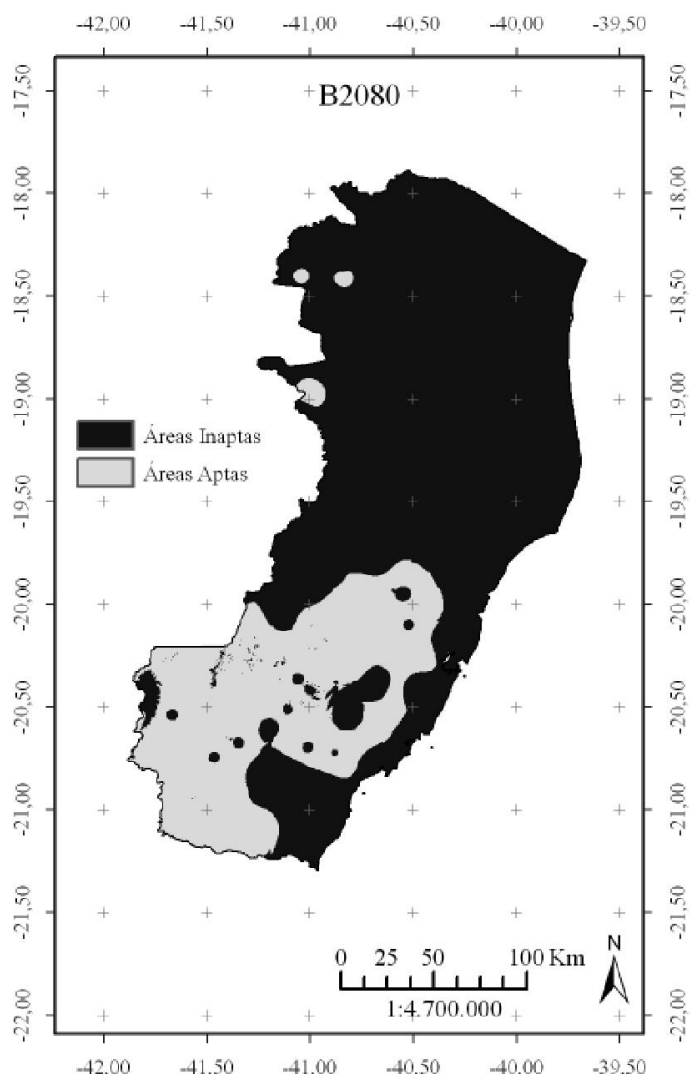

ASSAD, D. A. et al. Impacto das mudanças climáticas no zoneamento agroclimático do café no Brasil. Pesquisa Agropecuária Brasileira, Brasília, v. 39, n. 11, p. 1057-1064, 2004.

BERRY, P. M. et al. E. Assessing the vulnerability of agricultural land use and species to climate change and the role of policy in facilitating adaptation. Environmental Science \& Policy, v. 09, p. 189-204, 2006.

CARNEIRO, R. L. C. et al. Consumo de água em plantios de eucalipto - Parte 1: Determinação da condutância estomática em tratamentos irrigados e não irrigados. Revista Árvore, v. 32, p. 1-10, 2008.

CITYBRAZIL. Dados Gerais do Espírito Santo. Disponível em <http://www.citybrazil.com.br/es/geral.htm>. Acesso em: 5 de nov. 2009.

CUNHA, A. C. M. C. M. et al. Relações entre variáveis climáticas com produção e enraizamento de miniestacas de eucalipto. Revista Árvore, v. 33, n. 02, p. 195-203. 2009.

EWERT, F. et al. Future scenarios of European agricultural land use. I. Estimating changes in cop productivity. Agriculture, Ecosystems an Environment, v. 107, p. 101$116,2005$. 
FADER, M. et al. Virtual water content of temperate cereals and maize: Present and potential future patterns. Journal of Hydrology, v. 384, p. 218-231, 2010.

GOLFARI, L. Zoneamento ecológico do estado de Minas Gerais para reflorestamento. PNUD/FAO/IBDF BRA/71/545. (Série Técnica no 3) 65 p, 1975.

GORDON, C. et al. The simulation of SST, sea ice extents and ocean heat transports in a version of the Hadley Centre Coupled Model Without Flux Adjustments. Climate Dynamics, 16, p. 147-168, 2000.

HAIM, D.; SHECHTER, M.; BERLINER P. Assessing the impact of climate change on representative field crops in Israeli agriculture: a case study of wheat and cotton. Climatic Change, v. 86, p. $425-440,2008$.

IPCC - Intergovernmental Panel for Climate Change, Working Group I (IPCC-WGI): 2001. Climate change 2001 the Third Assessment Report: The Scientific Basis. Cambridge University Press, Cambridge.

IPCC - Intergovernmental Panel for Climate Change. Climate Change: Impacts, Adaptation, and Vulnerability. Cambridge University Press. 2001.

KADISHI, N.; KAN, I.; ZEITOUNI, N. Impacts of changes in annual rainfall distribution patterns on agriculture in Israel. Working Paper, Natural Resource and Environmental Research Center, Haifa University, Haifa. 2005.

LUO, Q. et al. Potential impact of climate change on wheat yield in South Australia. Agricultural and Forest Meteorology, Amsterdam, v. 132, p. 273-285, 2005.

MARTINS, F. B.; SILVA, J. C.; STRECK, N. A. Estimativa da temperatura-base para emissão de folhas e do filocrono em duas espécies de eucalipto na fase de muda. Revista Árvore, v. 31, n. 03, p. 373-381, 2007.

MIRANDA, J. R.; COUTINHO, A.C. Brasil Visto do Espaço. Campinas: Embrapa Monitoramento por Satélite. Disponível em: <http://www.cdbrasil.cnpm.embrapa.br>. Acesso em: 4 de nov. 2009.

NAPPO, M. E.; NAPPO, A. E.; PAIVA, H. N. Zoneamento Ecológico de Pequena Escala para Nove Espécies Arbóreas de Interesse Florestal no Estado de Minas Gerais. Revista Científica Eletrônica de Engenharia Florestal, v. 09, n. 03, p. 422-428, 2005.

PARRY, M.L. et al. Effects of climate change on global food production under SRES emissions and socio-economic scenarios. Global Environmental Change, v. 14, p. 53-67, 2004.

PEZZOPANE, J. E. M. et al. Espacialização da temperatura do ar no Espírito Santo. Revista Brasileira de Agrometeorologia, v. 12 , n. 01 , p. 151-158, 2004

PEZZOPANE, J. R. M. et al. Zoneamento de risco climático para a cultura do café Conilon no Estado do Espírito Santo. Revista Ciência Agronômica, v. 41, n. 03, p. 341-348, 2010.

ROUNSEVELL, M. D. A. et al. Future scenarios of European agricultural land use II. Projecting changes in cropland and grassland. Agriculture, Ecosystems and Environment, v. 107, p. 117-135, 2005.

THORNTHWAITE, C. W., Mather, R.J. The water Balance. New Gersey: Laboratory of Climatology, v. 08, 104 p, 1955.

TRABUCCO, A. et al. Climate change mitigation through afforestation/reforestation: A global analysis of hydrologic impacts with four case studies. Agriculture, Ecosystems and Environment. v. 126, p. 81-97. 2008. 